\title{
Die Verschwörung(en) hinter der Pandemie
}

\author{
Matthias Eder
}

Bill Gates genießt weltweite Bekanntschaft. Die Produkte der von ihm gegründeten Firma werden von Milliarden von Menschen täglich verwendet. Er wurde damit zu einem der reichsten Individuen der Welt. Doch mit dem Coronavirus verbreiteten sich auch einige erschreckende Behauptungen über den amerikanischen Unternehmer. Der Multimilliardär und seine Frau Melinda engagieren sich schon seit geraumer Zeit für Gesundheitsinitiativen, vor allem in Entwicklungsländern. Da ein Fokus der Arbeit ihrer Stiftung die Entwicklung und Verbreitung von Impfstoffen ist, waren Bill und Melinda Gates schon länger ein Feindbild in der Szene der Impfgegner:innen. Dem Ehepaar wird dabei jegliches karitatives Interesse abgesprochen, als Zweck der Stiftung und deren Projekte wird die Gier nach Profit oder Macht unterstellt. In einer besonders extremen Erzählung ginge es Bill Gates sogar darum, durch mittels Zwangsimpfungen verabreichter Gifte die Weltbevölkerung auf einige Hunderttausend zu reduzieren.

Während diese Narrative bisher außerhalb der Communities der Impfgegner:innen kaum bekannt waren, entstand durch die Pandemie eine neue Dynamik. Die Darstellung von Bill Gates als Profiteur oder sogar als Verursacher der Krise erreichte eine so weite Verbreitung, dass sich Zeitungen und Rundfunk genötigt sahen, derartige Behauptungen durch Faktenchecks zu widerlegen.

Besonders prägnant wird dies im hier abgebildeten Cartoon von Ben Garrison, einem US-amerikanischen politischen Karikaturisten, visualisiert (Abb. 22). Garrisons Zeichnungen, die häufig mit sexistischen, rassistischen und antisemitischen Motiven durchsetzt sind, sind vor allem in Teilen der amerikanischen Rechten, besonders der sogenannten Alt-Right, populär. Garrisons Bild trägt den Titel The Plannedemic, eine Kontraktion von «the planned pandemic», die geplante Pandemie.

Es zeigt Bill Gates, der sich vor einem dunklen Hintergrund über eine Schriftrolle beugt. Sein Gesicht ist zur teuflischen Fratze verzerrt, er blickt die Betrachtenden direkt an. Am Revers trägt er eine Totenkopfnadel. Die Schriftrolle beschreibt die sechs Schritte seines Plans: Unter der Ziffer eins ist eine Repräsentation des Virus zu sehen, darunter das Gesicht aus Ed- 


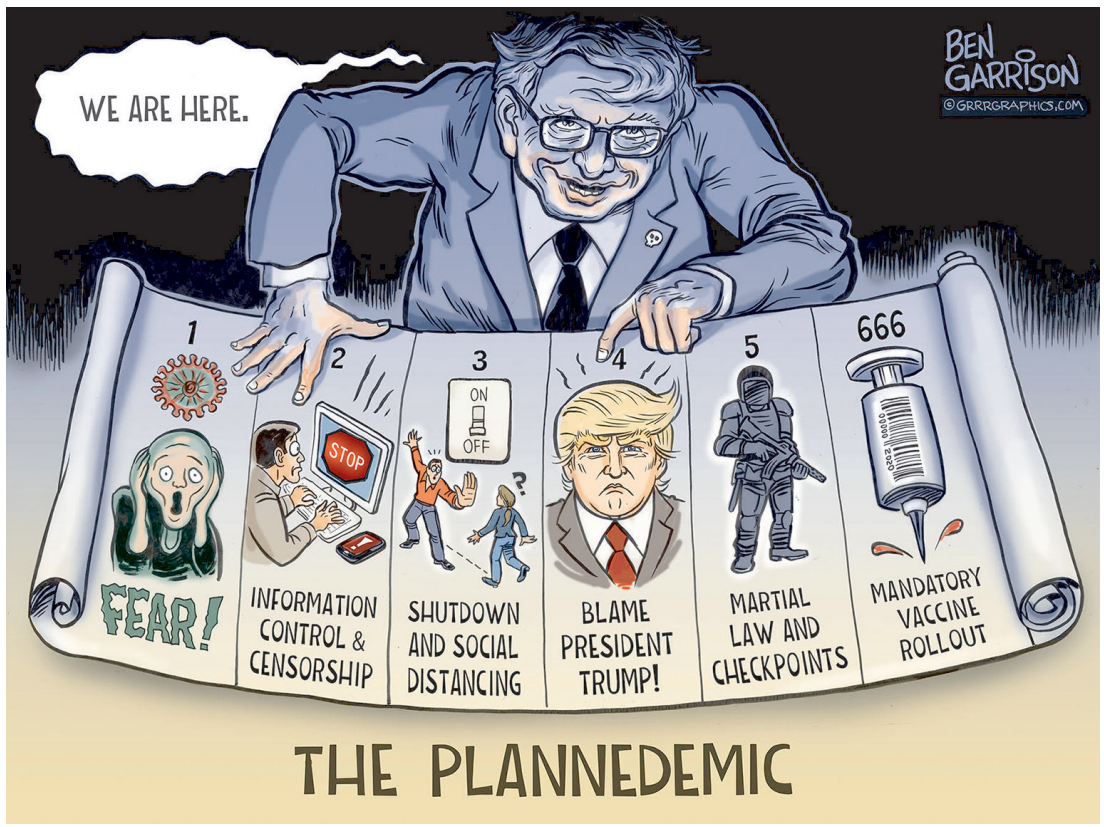

Abb. 22: Ben Garrison, The Plannedemic, 2020.

ward Munchs Gemälde Der Schrei und schließlich, in Großbuchstaben, das Wort «FEAR!». Die Bevölkerung soll also durch die Angst vor Covid-19 in einen Schockzustand versetzt werden. Stufe zwei zeigt einen Mann, der ungläubig auf einen Bildschirm starrt, auf dem ein großes Stoppschild prangt. Beschriftet ist sie mit «Informationskontrolle und Zensur». Als drittes folgt das Bild eines Schalters, der das Herunterfahren der Wirtschaft symbolisiert, und zweier Menschen, die sich nicht nahekommen dürfen und damit das Gebot der sozialen Distanzierung repräsentieren. Ziffer vier zeigt Donald Trump, dem die Schuld an der ganzen Situation in die Schuhe geschoben werden soll. Bei Punkt fünf sehen wir einen bewaffneten Menschen in Kampfmontur, "Kriegsrecht und Checkpoints», und schließlich eine riesige Spritze, die mit einem Barcode versehen ist, und für den Beginn von Zwangsimpfungen steht. Das letzte Feld ist mit 666 beschriftet.

Die Nachricht, die den Betrachtenden hier übermittelt werden soll, ist alles andere als subtil. Die Corona-Pandemie sei kein natürliches Phänomen, sondern Teil eines Plans, der von Bill Gates persönlich ausgeheckt worden sei, um eine globale Zwangsimpfung durchzusetzen. Zu seinen 
Beweggründen erfahren wir aus dem Bild selbst nur wenig. Die Spekulationen in den sozialen Medien reichen hier von reiner Profitgier, da Bill Gates ja an diversen Biotech-Unternehmen beteiligt ist, über die totale Überwachung durch mit der Impfung injizierter Mikrochips, bis hin zur einem von sogenannten globalen Eliten ersonnenen Plan zur Dezimierung der Menschheit, um so das Problem der Überbevölkerung ein für alle Mal zu lösen.

\section{Orthodoxe und heterodoxe Verschwörungstheorien}

Diese und ähnliche Narrative werden meist unter dem Begriff der Verschwörungstheorien zusammengefasst. Diese Bezeichnung hat allerdings im alltäglichen Sprachgebrauch einen stark wertenden Charakter. Eine Aussage als Verschwörungstheorie zu bezeichnen, bedeutet zumeist auch, ihr jeglichen Wahrheitsgehalt abzusprechen und so die Position des Gegenübers zu delegitimieren. Dies verschleiert jedoch die Tatsache, dass es in der Geschichte der Menschheit zahlreiche Verschwörungen gab, die durchaus historisch belegbar sind. Die Ermordung von Julius Caesar ist wohl eines der ältesten bekannten Beispiele.

Es gibt für historische Ereignisse aber teilweise konkurrierende Narrative. So ist beispielsweise auch die offizielle Version der Attentate vom 11. September 2001 die Geschichte einer Verschwörung, nämlich einer jihadistischen Verschwörung unter der Federführung von Osama bin Laden. Daneben existieren jedoch mehrere, von der gemeinhin akzeptierten Darstellung abweichende Erzählungen, die im öffentlichen Diskurs als Verschwörungstheorien bezeichnet werden, beispielsweise die Vermutung, die Attentate seien von der amerikanischen Regierung gewollt oder sogar selbst durchgeführt worden. Um diese verschiedenen Arten von Verschwörungsnarrativen klar zu trennen, verwenden die Soziologen Andreas Anton, Michael Schetsche und Michael Walter die Unterscheidung zwischen orthodoxen und heterodoxen Wirklichkeitskonstruktionen, also solchen, die dem offiziellen, von Regierungen oder anderen offiziellen Stellen bedienten Narrativ entsprechen, und solchen, die sich diesem entgegenstellen.

Mit dieser Unterscheidung ist keine Wertung verbunden, was den Wahrheitsgehalt bestimmter Aussagen anbelangt. Allerdings ist es durchaus so, dass viele populäre Verschwörungserzählungen, denen man heutzutage vor allem in den sozialen Medien begegnet, einer genaueren Überprüfung nicht standhalten. Man denke nur an die Theorie, dass die Erde eine Scheibe sei, was von Organisationen wie der NASA vertuscht werde, 
die sich in manchen Ecken des Internets noch immer großer Beliebtheit erfreut.

Im Falle des Narrativs, das die hier betrachtete Karikatur nahelegt, ist der Sachverhalt natürlich komplexer als bei der «Flachen Erde». Aber ich denke, wir können mit an Sicherheit grenzender Wahrscheinlichkeit annehmen, dass Bill Gates nicht wirklich plant, die Anzahl der Menschen durch vergiftete Impfungen auf wenige Hunderttausend zu reduzieren oder als Teil einer Weltregierung, der New World Order, die Macht auf dem Planeten zu übernehmen.

Einfache Erklärungen für komplexe Probleme

Der Erfolg solcher Erzählungen in einer scheinbar aufgeklärten Gesellschaft mag zunächst rätselhaft erscheinen, doch gibt es einige Faktoren, die ihre Verbreitung begünstigen. Eine Krise wie die Corona-Pandemie kann ein Gefühl von Ausgeliefertsein und Hilflosigkeit auslösen. Wir stehen einem unsichtbaren, übermächtigen Feind gegenüber, es gibt kaum Möglichkeiten, sich aktiv zur Wehr zu setzen. Noch dazu scheint das Virus fast aus dem Nichts aufgetaucht zu sein und hat binnen kürzester Zeit unser aller Leben, unser Wirtschaftssystem und auch das weltpolitische Geschehen auf den Kopf gestellt.

Bei solch gewaltigen Auswirkungen ist es schwierig zu akzeptieren, dass der Auslöser eine zufällige Mutation eines ansonsten harmlosen Erregers war, der bisher nur in Fledermäusen vorkam. Dieses mutierte Virus fand seinen Weg durch weitere Zufälle auf einen Markt in Wuhan, wo es dann den ersten Menschen infizieren konnte. Hier tritt ein psychologisches Phänomen auf, das bei der Entstehung und Verbreitung von Verschwörungserzählungen eine nicht zu unterschätzende Rolle spielt: Bei großen Ereignissen suchen Menschen nach großen Ursachen. Dieser proportionality bias genannte Effekt bewirkt, dass es oft leichter fällt, mächtige und im Verborgenen operierende Kräfte als Ursache einer Krise anzunehmen, als eine Verkettung unglücklicher Umstände.

Vor allem aber können Verschwörungserzählungen dazu beitragen, die Komplexität der Ereignisse zu reduzieren und sie in ein kohärentes Weltbild zu integrieren. Derartige Vorstellungen bieten eine Erklärung der Geschehnisse, in der nichts zufällig passiert, in der es eine klare Abgrenzung von Gut und Böse gibt und in der man zu einer privilegierten Gruppe von Menschen gehört, die Zugang zu einer Wahrheit haben, die von der Mehrheit noch nicht erkannt wurde. Es gibt ein klares Feindbild, gegen das demonstriert oder gekämpft werden kann. Man gehört zu den Erwachten. 
Durch die globale Vernetzung lassen sich schnell Gleichgesinnte finden. Solche digitale Neogemeinschaften, wie der Soziologe Andreas Reckwitz sie nennt, lassen eine Entwicklung von in sich geschlossenen Welterklärungen zu, die in der jeweiligen Filterblase sich selbst verstärkend zirkulieren können, ohne auf Dissens zu stoßen.

Die Gewissheit zu verstehen, was hinter dem Vorhang passiert und wer die wahren Strippenzieher sind, kann in einer Zeit der Krise das Gefühl vermitteln, sich aus der Passivität befreien zu können. Der Glaube, als einer von Wenigen die Wahrheit hinter den Dingen erkannt zu haben, führt zu einem Gefühl der Überlegenheit. In einer Gesellschaft, in der es zunehmend darum geht, das Individuelle und Singuläre der eigenen Person zu präsentieren, online wie offline, scheint die Mitgliedschaft in einer exklusiven Gemeinschaft der Wissenden umso erstrebenswerter.

\section{Die Suche nach den Schuldigen}

Erstaunlicherweise finden sich zwar neben der hier beschriebenen Verschwörungserzählung rund um Bill Gates noch eine Vielzahl anderer solcher Narrative mit wechselnden Protagonisten, es gibt allerdings keine klare Abgrenzung zwischen deren Anhänger:innen. So gibt es auch hierzulande mittlerweile eine wachsende Gefolgschaft der aus den USA stammenden QAnon-Bewegung, von den deutschsprachigen Medien gerne als «Verschwörungs-Sekte» bezeichnet, die in Donald Trump einen vom Militär installierten Retter sieht, der ein internationales Netzwerk von Satanist:innen und Pädophilen zerschlagen wird. Die Corona-Pandemie ist auch hier nur Teil eines großen Plans. Und obwohl es zunächst Widersprüche zwischen diesen verschiedenen Welterklärungen zu geben scheint, treten sie nicht in Konkurrenz zueinander, wie es bei religiösen Welterklärungen häufig der Fall ist. Hier werden die Narrative einfach integriert, mögliche Dissonanzen wegerklärt oder einfach ausgehalten. So hört man von den Gallionsfiguren der Szene, wie dem Popsänger Xavier Naidoo oder dem Kochbuchautor Attila Hildmann, oft ein Konglomerat aus Narrativen, die neben den bisher erwähnten noch Reichsbürgerideologie und antisemitische Vorstellungen transportieren.

Antisemitische Motive finden sich allerdings bei genauer Betrachtung in fast allen kursierenden Verschwörungserzählungen, in denen es um globale Eliten oder mächtige Einzelpersonen geht, die aus dem Schatten die Geschicke der Welt lenken. Häufig taucht in solchen Narrativen der jüdische Hedgefondmanager George Soros auf, dem bei seiner Unterstützung zahlreicher zivilgesellschaftlicher Projekte sinistre Motive unterstellt werden. 
Auch die jüdische Familie Rothschild, deren Mitglieder seit Generationen im Bankgewerbe tätig sind, wird gerne als schuldige Partei ausgemacht.

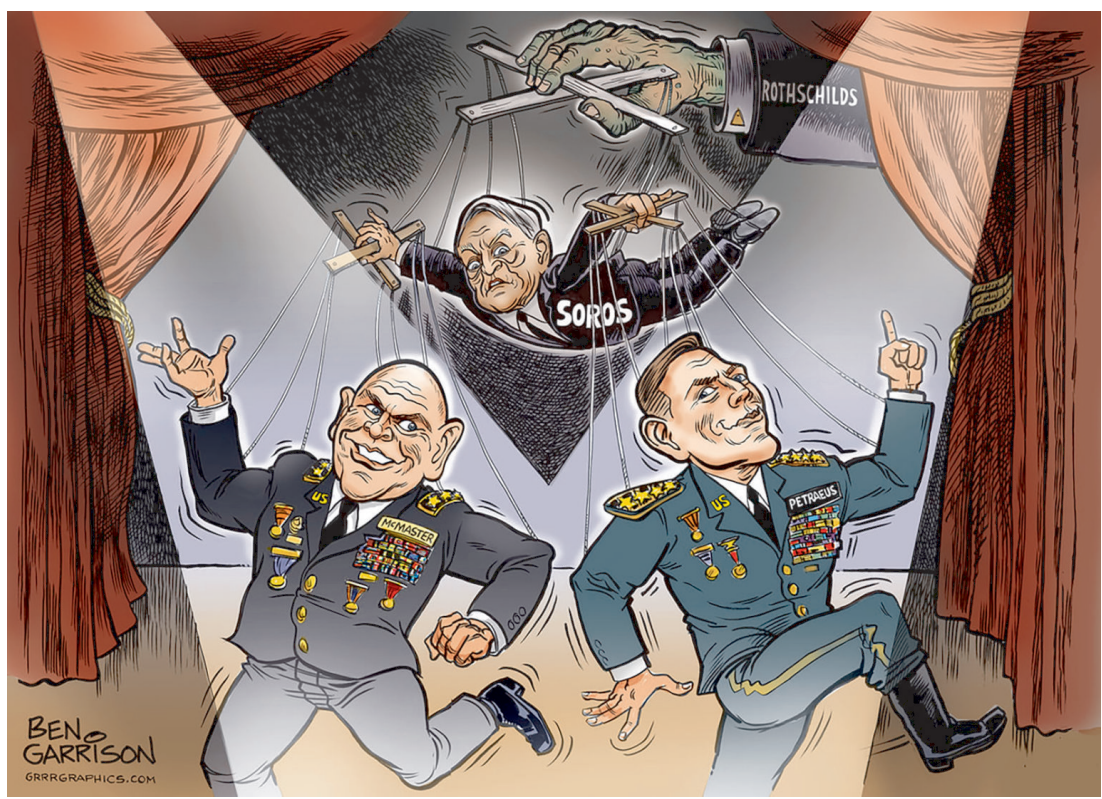

Abb. 23: Ben Garrison, McMaster's Masters, 2017.

Zur Veranschaulichung bietet sich eine weitere Karikatur von Garrison aus dem Jahre 2017 an (Abb. 23). Sie zeigt Soros als Puppenspieler. Er lenkt die an Fäden hängenden US-Generäle McMaster und Petraeus, die damals von der amerikanischen Rechten zu Antagonisten erklärt worden waren. Doch auch Soros ist fremdbestimmt, er ist selbst nur eine Marionette. Die Fäden zieht eine grünliche, faltige Hand. Der dazugehörige Arm, der sich aus dem Bereich hinter dem Vorhang ins Bild reckt, ist mit dem $\mathrm{Na}$ men «Rothschild» beschriftet. Das Schockierende an dieser Darstellung wird deutlich, wenn man sie mit antisemitischer Propaganda aus der Zeit des NS-Regimes vergleicht (Abb. 24). Die Darstellung des jüdischen Puppenspielers, der in dem hier abgebildeten Beispiel Churchill und Stalin kontrolliert, ist der in Garrisons Karikatur so ähnlich, dass es schwerfällt, hier an einen Zufall zu glauben. 


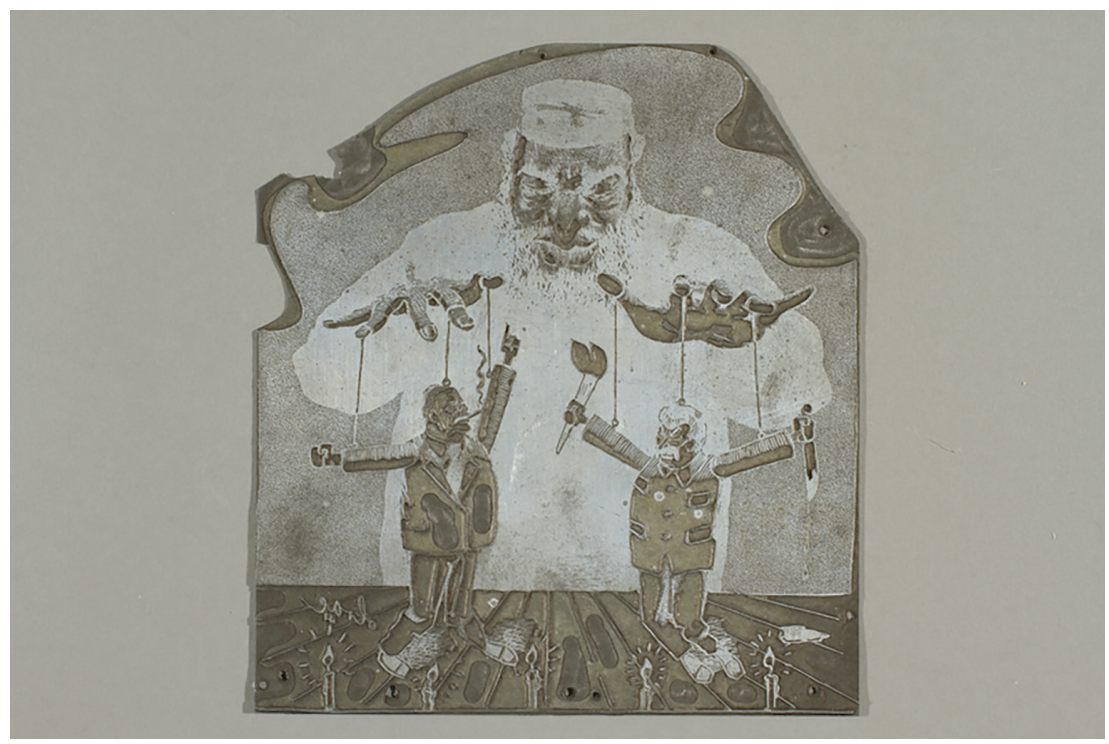

Abb. 24: Druckplatte mit antisemitischem Motiv, ca. 1941.

\section{Die Gefahr der Radikalisierung}

Bei den Protesten, die die Maßnahmen gegen die Corona-Pandemie begleiten, ist mittlerweile auch ein offener Bezug auf antisemitische Motive anzutreffen, die zunehmende Radikalisierung ist spürbar. Auf den sogenannten «Hygiene-Demos» vergleichen sich Gegner:innen der Maskenpflicht und Impfskeptiker:innen mit den Verfolgten des NS-Regimes. Die Rhetorik bei den «Corona-Rebellen» wird aggressiver, Journalist:innen werden als Verbreiter von Fake News angefeindet und sogar körperlich attackiert. Ein Protagonist der Protestbewegung, der bereits erwähnte Attila Hildmann, posiert gelegentlich mit der Reichskriegsflagge, die auf den Demonstrationen inzwischen allgegenwärtig ist. Er spricht von «globalen Eliten» und «Zionisten», gegen die mit allen Mitteln Widerstand geleistet werden müsse.

Es sind diese Dynamiken von zunehmender Aggressivität, simplen Erklärungsmustern und klaren Feindbildern, die den Glauben an Verschwörungen zu einer Gefahr für die Gesellschaft machen. Unter dem Deckmantel der Regierungskritik und der Wahrheitsfindung können sich derartige Vorstellungen im kollektiven Gedächtnis festsetzen, eine ewig fortgeschriebene Mythologie des Bösen im Verborgenen. Hat man die Prämissen 
dieser Narrative erst einmal akzeptiert, ist es nur noch ein kleiner Schritt, diese Vorstellungen auf unliebsame Gruppen zu lenken und diese als die wahren Mächte im Hintergrund allen Übels zu identifizieren. So konnte auch der Mord an 6 Millionen Juden und Jüdinnen durch die Nationalsozialisten mit uralten antisemitischen Narrativen legitimiert werden.

Die Vertreter:innen der beschriebenen Verschwörungstheorien werden häufig belächelt und als harmlose Spinner dargestellt. Doch wie hier gezeigt wurde, können solche Vorstellungen und Wirklichkeitsdeutungen den Weg für sehr viel gefährlicheres Gedankengut ebnen. Es ist daher zu begrüßen, dass sich Medien und Wissenschaft inzwischen ernsthaft mit diesem Themenkomplex auseinandersetzen. Eine produktive gesellschaftliche Auseinandersetzung setzt voraus, dass komplexe Vorgänge wie das Pandemiegeschehen verständlich erklärt, eingeordnet und vermittelt werden. Den Falschbehauptungen aus dem Lager der «Corona-Rebellen» hingegen muss entschieden durch Dekonstruktion und Aufklärung entgegengetreten werden. Die Pandemie ist eine Aufgabe historischer Ausmaße. Wir sollten sie bewältigen, ohne historische Fehler zu wiederholen.

\section{Literatur}

Anton, Andreas/Schetsche, Michael/Walter, Michael (Hg.), 2014, Konspiration. Soziologie des Verschwörungsdenkens, Wiesbaden: Springer VS.

Immhoff, Roland/Lamberty, Pia, 2020, A Bioweapon or a Hoax? The Link between Distinct Conspiracy Beliefs about the Coronavirus Disease (COVID-19) Outbreak and Pandemic Behavior, Social Psychological and Personality Science, 6.7.2020, https://doi.org/10.1177/1948550620934692 (aufgerufen am 1.8.2020).

Reckwitz, Andreas, 2017, Die Gesellschaft der Singularitäten, Berlin: Suhrkamp.

Simonsen, Kjetil Braut, 2020, Antisemitism and Conspiracism, in: Butter, Michael/ Knight, Peter (Hg.) Routledge Handbook of Conspiracy Theories, London: Routledge, 357-370. 